\title{
Design and Analysis of Nonuniformly Shaped Heaters for Improved MEMS-Based Electrothermal Displacement Sensing
}

\author{
Anthony G. Fowler, Student Member, IEEE, Ali Bazaei, Member, IEEE, and S. O. Reza Moheimani, Fellow, IEEE
}

\begin{abstract}
Conventional heaters used in microelectromechanical systems (MEMS) electrothermal displacement sensors typically feature a uniform cross section, which results in a nonuniform temperature profile. In this paper, electrothermal sensors with a shaped beam profile are introduced, with simulation results showing that a much flatter temperature distribution is achieved across the length of the heater. The proposed sensor design is implemented as the displacement sensor for a MEMS nanopositioner together with a more conventional electrothermal sensor design for comparative purposes. Experimental testing indicates that the shaped profile significantly improves upon the conventional sensor design in a number of areas, including sensitivity, linearity, and noise performance.

[2012-0291]
\end{abstract}

Index Terms-Displacement sensor, microelectromechanical systems (MEMS) electrothermal sensing, silicon thermal heaters.

\section{INTRODUCTION}

$\mathbf{I}$ $\mathrm{N}$ A microelectromechanical systems (MEMS)-based device where microactuators are used to produce mechanical displacements, embedded or external position sensors are often used to enable the closed-loop feedback control of the system. Common displacement sensing methods used for MEMS devices include capacitive sensing, electrothermal sensing, piezoelectric sensing, piezoresistive sensing, and optical microscopy [1]. Of these technologies, electrostatic and electrothermal sensors are arguably the simplest to integrate into a fabricated MEMS device as they can be constructed from the same silicon layer that often forms the main structure of the mechanical system.

Embedded capacitive sensors are commonly employed in MEMS devices and have been demonstrated to be able to provide position measurements with subnanometer precision [2]. However, the comb-finger capacitors that generally comprise these capacitive sensors tend to occupy large areas of the MEMS die, and therefore, their integration into MEMS devices can be difficult when die space is constrained.

In contrast, electrothermal sensors have been demonstrated to be able to provide subnanometer precision using a compar-

Manuscript received October 8, 2012; revised December 6, 2012; accepted January 4, 2013. Date of publication February 4, 2013; date of current version May 29, 2013. This work was supported by the Australian Research Council. Subject Editor N. F. de Rooij.

The authors are with the School of Electrical Engineering and Computer Science, The University of Newcastle, Callaghan, N.S.W. 2308, Australia (e-mail: Anthony.Fowler@uon.edu.au; ali.bazaei@newcastle.edu.au; Reza.Moheimani@newcastle.edu.au).

Color versions of one or more of the figures in this paper are available online at http://ieeexplore.ieee.org.

Digital Object Identifier 10.1109/JMEMS.2013.2240261 atively compact design, while maintaining a dynamic range of hundreds of micrometers at bandwidths of up to $10 \mathrm{kHz}$ [3] and being less prone to high-frequency noise [4]. For such reasons, integrated electrothermal displacement sensors continue to be demonstrated in a growing number of MEMS applications [5]-[11].

An early implementation of electrothermal displacement sensing was in a silicon accelerometer, where thermopiles were utilized to detect temperature differences between a heat source and a seismic mass acting as a heat sink [12].

In [13], the concept is applied in a data-storage mechanism based on an atomic force microscope cantilever with a heated tip. In this implementation, the distance between the heater cantilever and the storage substrate is measured by monitoring the resistance of the heater. Changes in the spacing between the heater and the substrate vary the conduction of heat between the two bodies, resulting in changes in the heater temperature that can be measured due to the temperature-dependent resistance of the heater cantilevers. Further analysis of the dynamics of this type of microheater is presented in [14]. In [15], the use of electrothermal sensors for displacement measurement is extended to the position sensing of a MEMS scanner containing the data-storage medium.

A micromachined electrothermal sensor consisting of a pair of resistive heaters operated in a differential configuration is presented in [16]. The sensor is fabricated via a single-layer MEMS process and is used for the in-plane displacement measurement of a thermally actuated MEMS nanopositioner. A more detailed analysis of the electrothermal sensor and actuator is presented in [5], [17], and [18].

A similar single-layer device containing a movable stage and in-plane electrothermal displacement sensors is presented in [19], where a comparison between applying a constant bias current and a constant bias voltage to the sensor is also made.

The thermal and electrical properties of a single silicon heater are analyzed in [20]; however, as noted in [3], [14], and [21], the dynamic range, linearity, and drift associated with electrothermal sensing can be significantly improved by operating two heaters in a differential configuration.

One characteristic of the heaters used in electrothermal sensors is that the spatial distribution of temperature tends to be highly nonuniform, with the maximum temperature being located at the center of the heater (as shown in Fig. 1) and the minimum temperatures being found at the two ends of the heater [5], [20]. This means that the sensitivity of the sensor, which increases with temperature, is therefore limited by the 


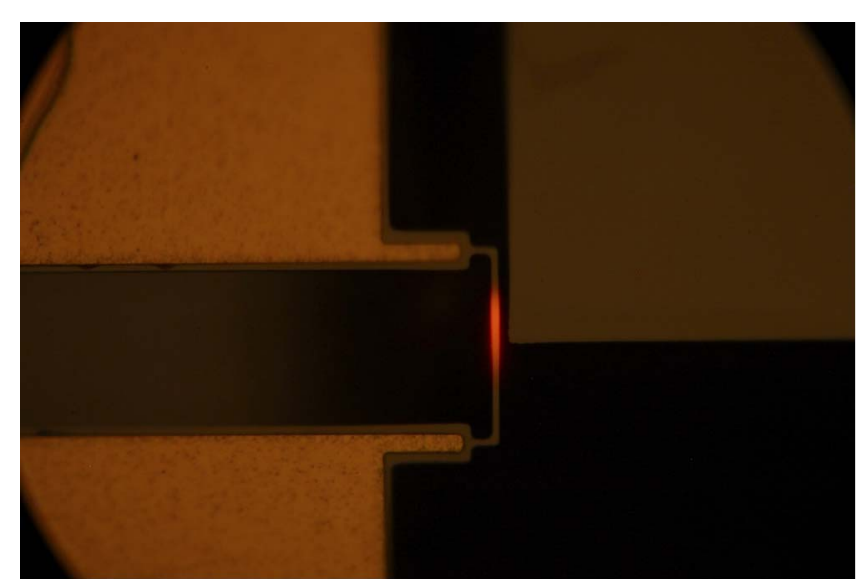

Fig. 1. Visible-light photograph showing heated central area of electrothermal heater.

temperature of the central area of the heater. In addition, the nonuniform temperature profile can be a source of nonlinearity that limits the dynamic range and linear operation of the sensor.

The same nonuniform temperature profiles have also been shown to limit both the static and dynamic performance of electrothermal actuators [22]. Significant performance improvements can be made through the use of actuation beams with a nonuniform cross section, yielding a much more uniform spatial temperature distribution [22]-[25].

This paper proposes that the performance of electrothermal sensors can be similarly improved by using heaters with a nonuniform profile in order to achieve a flatter temperature distribution. The new heater design is assessed through implementation as an electrothermal displacement sensor for a MEMS-based nanopositioner. A second electrothermal sensor featuring heaters with a conventional profile is also included in the device for comparison purposes.

In Section II of this paper, the profile of the nonuniform heater for improved electrothermal sensing is introduced. The resulting temperature profile of the proposed heater is predicted in Section III, with a mathematical analysis from first principles being used to model the temperature distribution and assess the effect of the applied electrical bias. Section IV describes the design and fabrication of the MEMS nanopositioner used to test the proposed heater and compare its performance with a more conventional uniformly shaped heater. The results from experimental testing performed on the fabricated MEMS device are discussed in Section V, with a summary of the work being presented in Section VI.

\section{Proposed Heater Profile}

A nonuniform profile for the sensing heaters is proposed, as shown in Fig. 2(a). For comparative purposes, a conventional electrothermal sensor with uniformly shaped heaters is fabricated on the same silicon-on-insulator MEMS die, as shown in Fig. 2(b).

For a heater with length $L$, the proposed beam profile is given by the following formula:

$$
w(x)=b+a \tanh \left[\alpha\left(x_{0}-|x|\right)\right]
$$

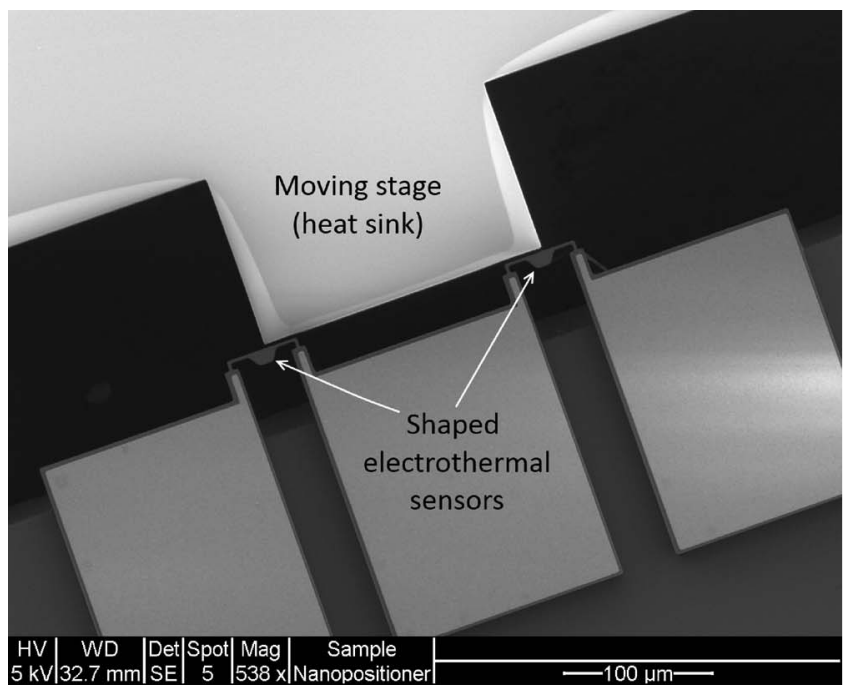

(a)

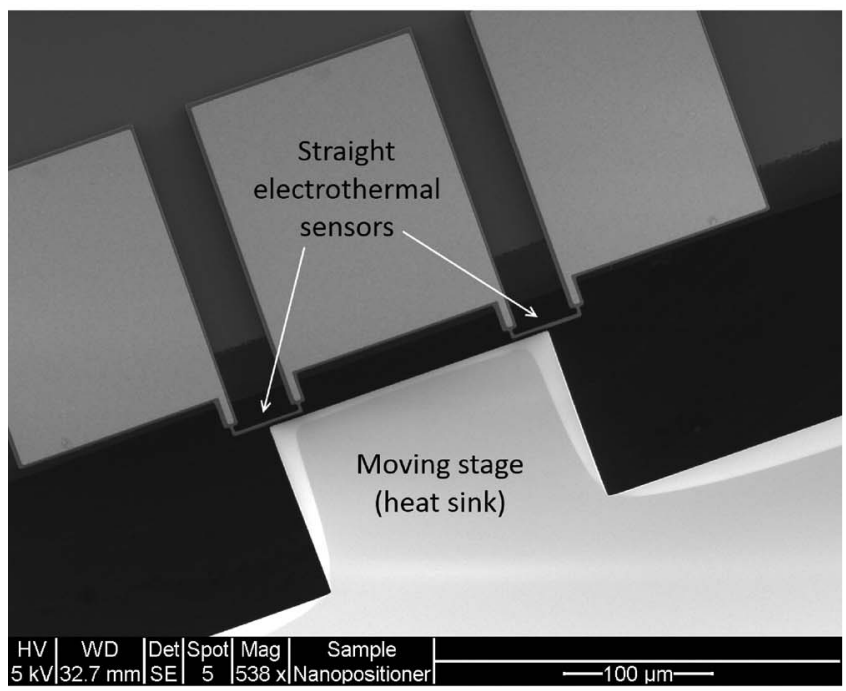

(b)

Fig. 2. (a) SEM image of curved sensors. (b) SEM image of straight sensors.

where $x$ is the coordinate along the beam as measured from the center, $x_{0}$ and $\alpha$ are parameters affecting the profile of the heater, and $a$ and $b$ are obtained from (1) in terms of the desired maximum width $w_{1}$ and minimum width $w_{0}$, given by

$$
\left[\begin{array}{l}
b \\
a
\end{array}\right]=\left[\begin{array}{cc}
1 & \tanh \left[\alpha\left(x_{0}-L / 2\right)\right] \\
1 & \tanh \left(\alpha x_{0}\right)
\end{array}\right]^{-1}\left[\begin{array}{l}
w_{0} \\
w_{1}
\end{array}\right] .
$$

A hyperbolic form was chosen to allow a smooth transition in the width of the beam. The use of a tanh function allows the heater profile to be defined with a unique formula having a minimum number of parameters, which simplifies the process of analyzing the effect of factors such as the width of the thicker section of the heater on the temperature distribution. It is also flexible enough to approximate almost any desired symmetric beamwidth profile that gradually decreases from the center to the ends of the heater. The concise expression (1) also avoids the use of multiple expressions for different sections of the beam.

Using the aforementioned parameters, a range of shaped heater profiles was modeled and simulated using various values 


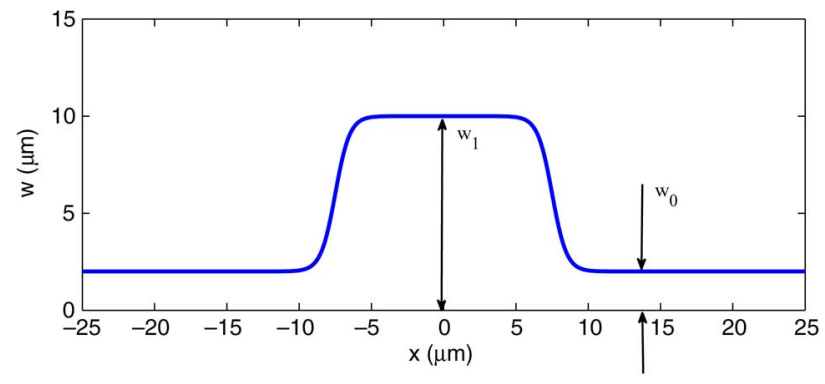

Fig. 3. Profile of proposed curved sensor with length $L=50 \mu \mathrm{m}$.

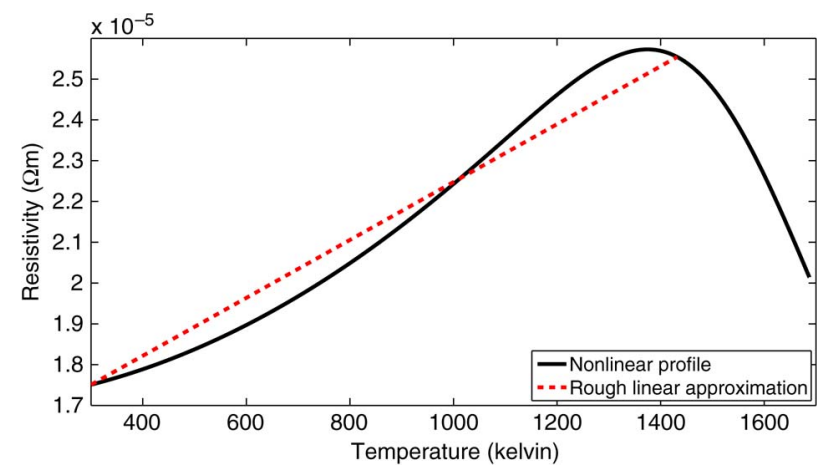

Fig. 4. Electrical resistivity versus temperature for silicon heaters.

of $w_{1}, \alpha$, and $x_{0}$. The room-temperature heater resistance and $L$ were assumed to remain constant, while $w_{0}$ was chosen based on the limitations of the fabrication process. The shaped profile presented in this paper uses the values $x_{0}=0.15 \mathrm{~L}$ and $\alpha=50 / \mathrm{L}$, which were found to yield an optimal result in that the profile provides a wide area with uniform temperature while avoiding the use of an excessively thick heater, which has implications for the bandwidth of the sensor as discussed later in this paper. The resulting profile of the shaped sensor is shown in Fig. 3.

\section{Sensor Analysis and Simulation Results}

Prediction of the electrothermal sensor temperature was performed by solving a 1-D heat equation that includes the nonlinear effects of spatial variations of the beamwidth and the thermal dependence of electrical resistivity of doped silicon. To concentrate on how the proposed beamwidth profile affects the uniformity of the temperature profile compared with that of the uniform-width heater, the effect of the nonuniform proximity of the heat sink is ignored. Using the method described in [20], the nonlinear temperature dependence of resistivity is taken into account with a dopant concentration of $8 \times 10^{19} \mathrm{~cm}^{-3}$. Fig. 4 shows the resulting temperature profile of resistivity, which is used in the subsequent analysis.

To reduce the heat equation to one dimension, it was assumed that the beam temperature and electrical current density are both uniform in the transverse direction. This allows the following differential equation to be obtained as the heat equation:

$\frac{I^{2}}{A_{e}(x)} \rho_{e}(\widetilde{T})+k \frac{\partial}{\partial x}\left[A(x) \frac{\partial \widetilde{T}}{\partial x}\right]=K_{a}(x) \widetilde{T}(x)+A(x) c \rho \frac{\partial \widetilde{T}}{\partial t}$ where $\widetilde{T}(x, t)=T(x, t)-T_{a}$ is the temperature increase at position $x$ and time $t$ with respect to the ambient temperature $T_{a} ; \rho_{e}$ is the electrical resistivity, which depends on temperature according to Fig. $4 ; A_{e}(x)=(2 \mu \mathrm{m}) w(x)$ is the effective crosssectional area through which constant electric current $I$ flows; $k=130(\mathrm{~W} / \mathrm{Km})$ is the thermal conductivity of the heater; $\rho$ is the density of the heater material; $c$ is the specific heat capacity of the heater material; $A(x)=(25 \mu \mathrm{m}) w(x)$ is the cross-sectional area of the heater; and $K_{a}(x)$ is the thermal conductance per unit length between the heater and the ambient environment at $x$, which is assumed to increase with beamwidth as

$$
K_{a}(x)=0.03 \times \frac{w(x)+25 \mu \mathrm{m}}{27 \mu \mathrm{m}},\left(\frac{\mathrm{W}}{\mathrm{Km}}\right)
$$

where $w$ is in micrometers. Under stationary conditions, the heat equation can be simplified as

$$
\frac{I^{2}}{A_{e}(x)} \rho_{e}(\widetilde{T})+k \frac{d}{d x}\left[A(x) \frac{d \widetilde{T}}{d x}\right]=K_{a}(x) \widetilde{T}(x) .
$$

The beam profile is symmetric with respect to the center of the sensor $x=0$, and as are the end point boundary conditions at $x= \pm(L / 2)$. This means that the temperature profile is symmetric with respect to the center of the sensor and that the temperature gradient is zero at $x=0$. The solution interval can therefore be halved $(x \in[0,(L / 2)])$ by applying the following boundary conditions:

$$
\left.\frac{d \widetilde{T}}{d x}\right|_{x=0}=0,\left.\left[G \widetilde{T}(x)+k A(x) \frac{d \widetilde{T}}{d x}\right]\right|_{x=\frac{L}{2}}=0
$$

where $G$ refers to the total heat conductance between each end point of the heater and the ambient environment. This conductance is higher due to the connection to a gold-covered pad and is assumed to be proportional to the size of the cross section of the heater at the end point, as

$$
G=35 \times 10^{-6} w_{0},\left(\frac{\mathrm{W}}{\mathrm{K}}\right)
$$

where $w_{0}$ is in micrometers. To solve the two-point boundary value problem (5) and (6) in MATLAB, the numerical solver bvp $4 c$ was used, which requires an initial guess for the solution. As the differential equation is nonlinear, the problem may not have a unique solution. To ensure that the solver converges to the desired solution, a rough approximation of the temperature profile (described in Section III-A) was used as the initial guess.

Fig. 5 shows the simulated temperature profiles of both the proposed and conventional heaters with a length of $50 \mu \mathrm{m}$. These sensors use the parameters shown in Table I, with both sensors having very similar resistances. From this simulation, it is clear that the proposed shaped heater profile results in a more uniform temperature distribution compared to the conventional straight heater. In addition, the shaped sensor requires a smaller bias voltage to achieve a similar temperature range as the straight sensor. 


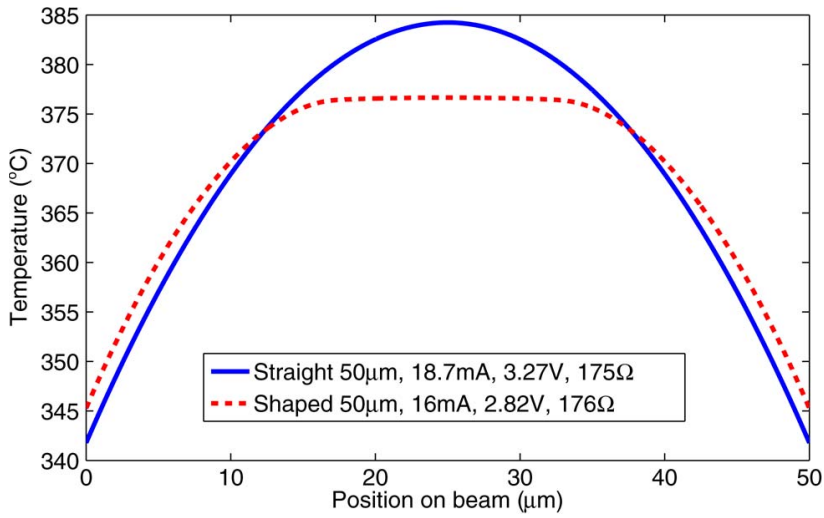

Fig. 5. Simulated temperature profiles of the electrothermal sensor heaters (excluding the heat sink effect for simplicity).

TABLE I

SENSOR Parameter Values

\begin{tabular}{|c|c|c|c|c|}
\hline Parameters & $\begin{array}{c}w_{0} \\
(\mu \mathrm{m})\end{array}$ & $\begin{array}{c}w_{1} \\
(\mu \mathrm{m})\end{array}$ & $\begin{array}{c}I \\
(\mathrm{~mA})\end{array}$ & $\begin{array}{c}\text { Voltage } \\
(\mathrm{V})\end{array}$ \\
\hline Shaped-Simulation & 2 & 10 & 16 & 2.82 \\
\hline Straight-Simulation & 2.75 & 2.75 & 18.7 & 3.27 \\
\hline Shaped-50 $\mu \mathrm{m}$ & 2 & 10 & 21.57 & 6.1 \\
\hline Straight-50 $\mu \mathrm{m}$ & 2 & 2 & 16.4 & 6.1 \\
\hline Shaped-30 $\mu \mathrm{m}$ & 2 & 10 & 20.55 & 3.2 \\
\hline Straight- $30 \mu \mathrm{m}$ & 2 & 2 & 17.33 & 3.23 \\
\hline
\end{tabular}

\section{A. Rough Approximation}

To obtain an approximation of the shaped heater's temperature profile to be used as an initial guess for the numerical solver, an imaginary heater with uniform beamwidth was considered. This heater was considered to have a similar length as the shaped heater and to have the same resistance as the shaped heater at room temperature. Moreover, for temperatures in the range of $[300,1400] \mathrm{K}$, we assume the following linear dependence for resistivity, as shown in Fig. 4:

$$
\rho(\widetilde{T}) \approx \rho_{0}\left(1+\alpha_{0} \widetilde{T}\right)
$$

where $\rho_{0}=1.75 \times 10^{-5} \Omega \mathrm{m}$ and $\alpha_{0}=4.0548 \times 10^{-4} \mathrm{~K}^{-1}$. Using the methodology described in [18], the solution of the boundary value problem (5) and (6) can be summarized in the following form:

$$
\widetilde{T}= \begin{cases}\frac{\gamma}{\beta^{2}}\left[1-\frac{\cosh (\beta x)}{\left.\cosh \left(\beta \frac{L}{2}\right)+\frac{k A \beta}{G} \sinh \left(\beta \frac{L}{2}\right)\right]},\right. & \text { if }: I<I_{c} \\ \frac{\gamma}{\beta^{2}}\left[\frac{\cos (\beta x)}{\cos \left(\beta \frac{L}{2}\right)-\frac{k A \beta}{G} \sin \left(\beta \frac{L}{2}\right)}-1\right], & \text { if: } I>I_{c} \\ \frac{k A \gamma L}{2 G}+\frac{\gamma}{2}\left[\left(\frac{L}{2}\right)^{2}-x^{2}\right], & \text { if: } I=I_{c}\end{cases}
$$

where

$$
\begin{aligned}
\beta & =\sqrt{\frac{\alpha_{0} \rho_{0}}{k A_{e} A}\left|I^{2}-I_{c}^{2}\right|} \\
I_{c} & =\sqrt{\frac{K_{a} A_{e}}{\alpha_{0} \rho_{0}}} \\
\gamma & =\frac{I^{2} \rho_{0}}{k A_{e} A} .
\end{aligned}
$$

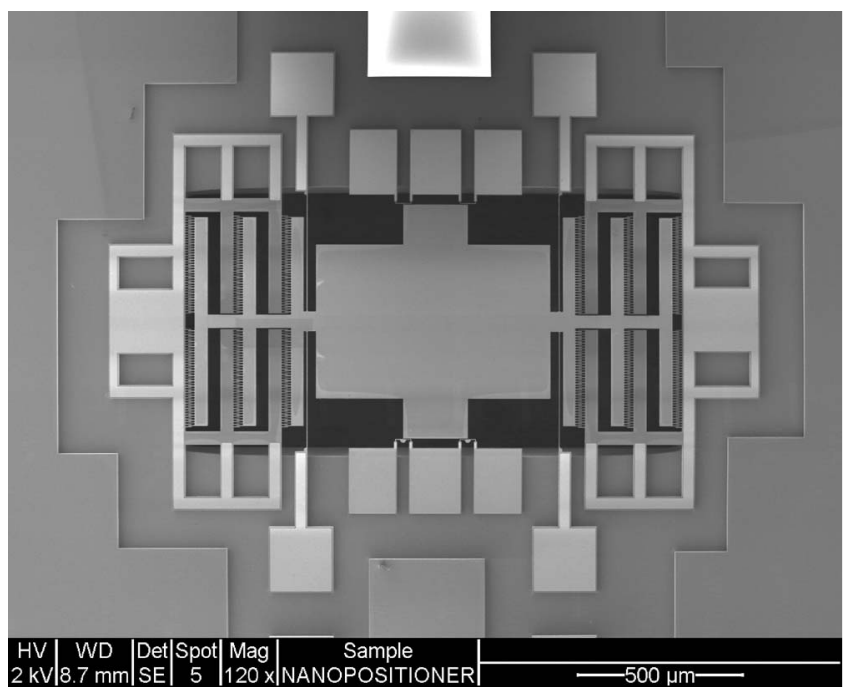

Fig. 6. SEM image of fabricated MEMS nanopositioner with integrated electrothermal sensors.

\section{FABRICATION AND EXPERIMENTAL SETUP}

For testing and characterization purposes, the proposed electrothermal sensor (consisting of a pair of shaped heaters) was implemented as an integrated displacement sensor for a onedegree-of-freedom MEMS nanopositioner which uses electrostatic comb-drive actuators in a bidirectional configuration. This nanopositioner has been previously tested and characterized using straight electrothermal sensors [4], allowing this device to serve as a useful platform for assessing the performance of the new shaped sensor design. For direct comparison purposes, an electrothermal sensor comprising the more conventional straight heaters was implemented on the opposite side of the nanopositioner stage. The MEMS device was fabricated using the silicon-on-insulator-based SOIMUMPs process provided by MEMSCAP, with the main features of the device including actuators, stage, and electrothermal sensors being fabricated from $25-\mu \mathrm{m}$-thick doped silicon [26]. A scanning electron microscope (SEM) image of the fabricated device is shown in Fig. 6.

During operation, each pair of electrothermal sensors experiences differential changes in resistance due to the displacement of the nanopositioner stage. These changes in resistance are converted into an output voltage via the circuit shown in Fig. 7. When a constant dc bias voltage is applied across each sensor, variations in the resistance of each sensor due to a displacement of the stage result in a small change in the current flowing through each sensor. These currents are converted into voltages via separate transimpedance amplifiers which have a gain close to 1 . Finally, an instrumentation amplifier is used to take the difference of these signals and amplify this differential signal with a gain of approximately $90 \mathrm{~V} / \mathrm{V}$, rejecting the commonmode component of both sensors. In this manner, the effect of factors such as the ambient temperature and sensor aging on the displacement measurement is greatly reduced. Such a differential sensing scheme also helps to improve overall sensor linearity and reduce output drift [16]. 


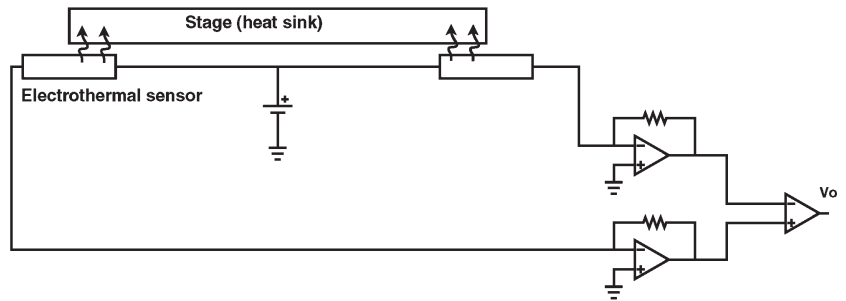

(a)

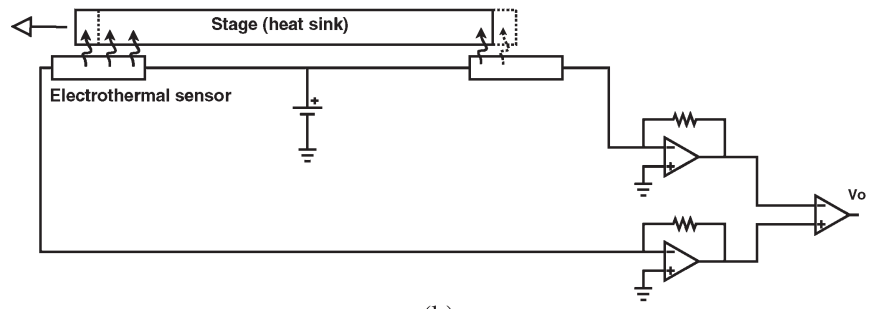

(b)

Fig. 7. Schematic diagram of thermal sensing mechanism. (a) Stage in neutral position. (b) Stage displaced due to actuation.

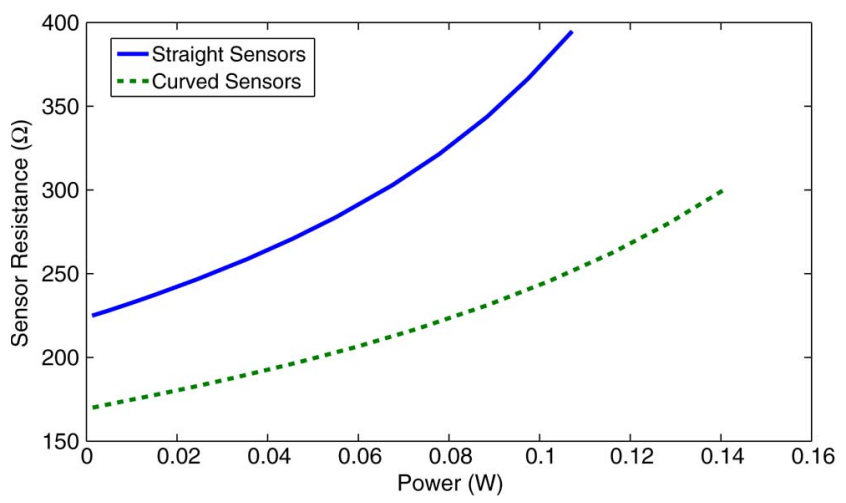

Fig. 8. Voltage and current characteristics of $50-\mu \mathrm{m}$ sensors.

\section{EXPERIMENTAL RESULTS}

By varying the bias voltage applied to each electrothermal sensor and measuring the current flowing through each heater, the relationship between the power dissipated by each heater and its resistance was obtained, as shown in Fig. 8. Assuming that the change in the temperature of the heater is linearly proportional to the power dissipated by the beam, Fig. 8 depicts the highly temperature-dependent resistivity of the doped silicon that comprises the heaters. The increasing curvature of the plots in Fig. 8 with respect to input power shows that the temperature dependence of the silicon's resistivity becomes more nonlinear at higher temperatures.

The relationship between the sensor output and the displacement of the nanopositioner stage was obtained using a Polytec MSA-400 microsystem analyzer (MSA). A series of voltage steps of varying amplitudes was applied to the nanopositioner's electrostatic actuators, with the displacement of the stage being measured using the MSA's pattern recognition algorithms. The output of the sensor circuit was simultaneously recorded, allowing the static characteristic of sensor output versus stage displacement to be obtained, as shown in Fig. 9. The parameters of the $50-\mu \mathrm{m}$ sensors used to obtain these data are shown in Table I. Both the straight and curved sensors were supplied with

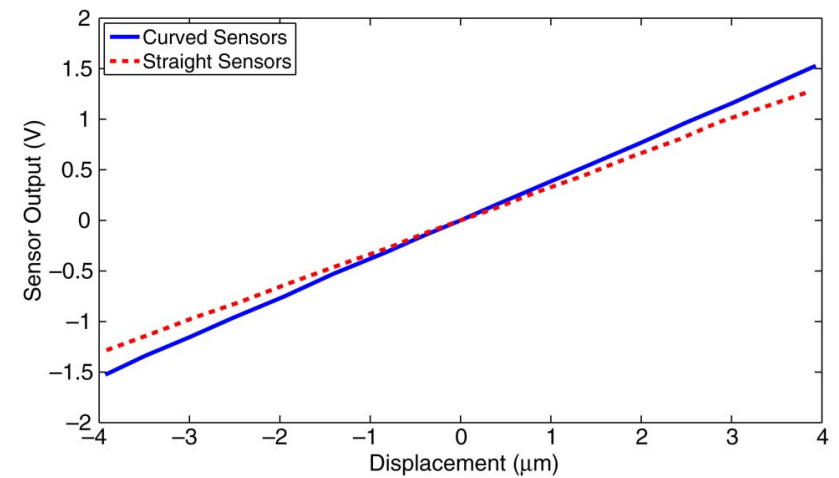

Fig. 9. Sensor output versus stage displacement for $50-\mu \mathrm{m}$ curved and straight sensors.

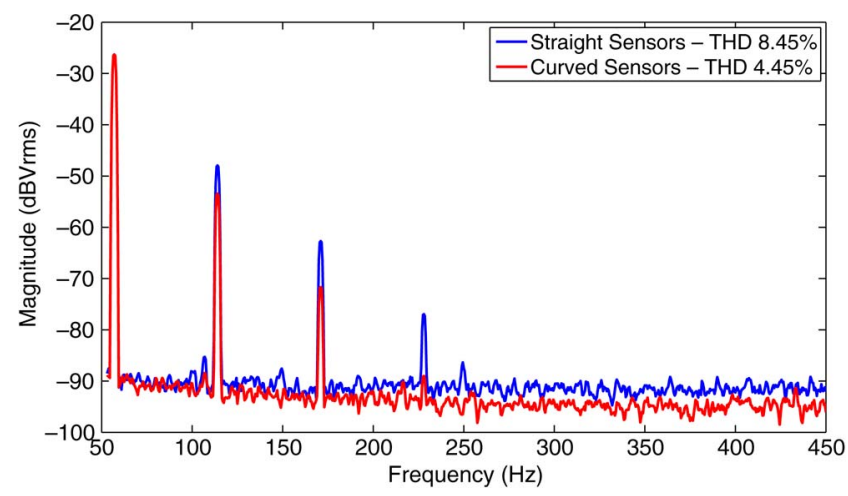

Fig. 10. Frequency-domain analysis of $30-\mu \mathrm{m}$ sensor outputs with sinusoidal stage actuation.

a dc bias of $6.1 \mathrm{~V}$. This has been experimentally determined to result in temperatures close to the melting point of the silicon forming the sensors and so represents the maximum sensitivities attainable. From the graph, it is evident that the shaped sensor provides a higher sensitivity than the straight sensor when using the same bias voltage.

The linearity of the sensors with respect to the stage displacement was assessed by measuring the frequency-domain output of the sensing circuit. As the maximum displacement attainable in one direction by the nanopositioner is limited to around $5 \mu \mathrm{m}$, this test utilized sensors with shorter beam heaters of length $30 \mu \mathrm{m}$. The parameters for these heaters are shown in Table I. A $57-\mathrm{Hz}$ sinusoidal signal, with an amplitude of $12.5 \mathrm{~V}$ superimposed on a $32.5-\mathrm{V}$ dc bias, was supplied to the nanopositioner's electrostatic actuator. The biases applied to the straight and curved sensors were then adjusted such that the magnitudes of the peak shown on the signal analyzer at the fundamental actuating frequency were matched. While the nanopositioner's electrostatic actuators also possess a nonlinear characteristic due to the quadratic relationship between voltage and actuation force [27], [28], this test was performed with the assumption that any assessed difference in linearity is purely due to the influence of the sensors. The signal analyzer output, shown in Fig. 10, indicates that the magnitudes of the higher order harmonics in the curved sensor output are measurably lower than that in the output of the straight sensors. The total harmonic distortion (THD) figures were measured to be $8.45 \%$ for the straight sensors and $4.43 \%$ for the curved sensors-this 


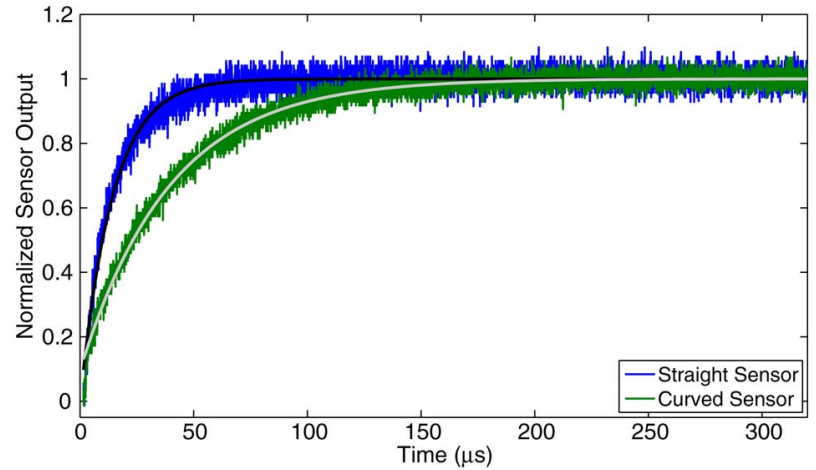

(a)

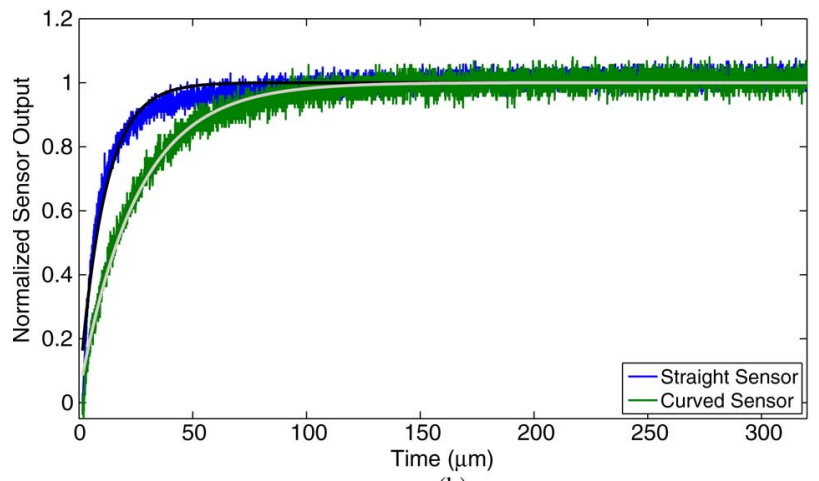

(b)

Fig. 11. Response of curved and straight sensors to a step change in voltage bias. (a) $50-\mu \mathrm{m}$ sensors. (b) $30-\mu \mathrm{m}$ sensors.

significant difference implies that the curved sensors have a more linear response. It should be noted that a portion of this distortion stems from the nonlinear behavior of the actuator-hence, it cannot be expected that the output distortion would vanish even if the sensor were perfectly linear.

In addition, the noise floor of the curved sensor is shown in Fig. 10 to be at a lower magnitude at higher frequencies than that of the straight sensor. With the actuator inputs then electrically grounded, the power spectral density (PSD) of the outputs of each sensor was measured and compared. These measurements indicate that the PSD of the curved sensors approaches $4 \mu \mathrm{V} / \sqrt{\mathrm{Hz}}$, which is equivalent to $0.04 \mathrm{~nm} / \sqrt{\mathrm{Hz}}$. In comparison, the PSD of the straight sensors approaches $8.60 \mu \mathrm{V} / \sqrt{\mathrm{Hz}}$, which is equivalent to $0.08 \mathrm{~nm} / \sqrt{\mathrm{Hz}}$.

To assess the dynamic behavior of each of the electrothermal sensors, it was determined that the sensor bandwidths could not be accurately assessed via the actuation of the nanopositioner stage due to its own bandwidth being limited. Instead, a single heater from each electrothermal sensor was supplied with a small step change in its electrical bias voltage of approximately $0.1 \mathrm{~V}$, with an amplified version of the resulting response at the output of its corresponding transimpedance amplifier being used to assess the dynamic performance of the sensor. As noted in [3], the application of such a step change in the electrical bias results in an initial change in the sensor output due to the change in applied voltage, followed by a slower variation resulting from the changing sensor resistance due to the change in heater temperature. It is this second response that is representative of the effective dynamics of the sensor and which is shown in Fig. 11(a) for both straight and curved electrothermal sensors

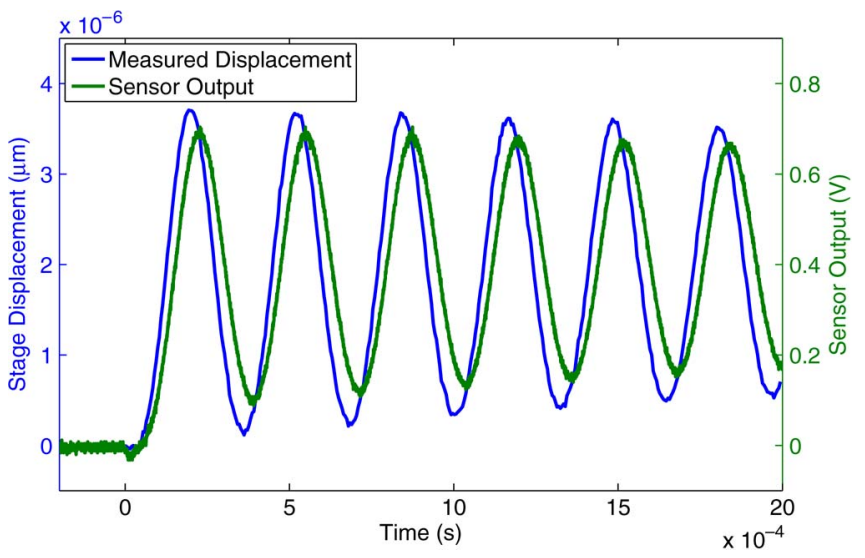

Fig. 12. Comparison of step response outputs from MSA and curved electrothermal sensor.

with the length of $50 \mu \mathrm{m}$. As the time constants of the sensor outputs are the main characteristics of interest, each of the sensors' step responses has been fitted with a first-order exponential function and normalized. As shown, the straight sensor's response has a time constant of $14.2 \mu \mathrm{s}$, while the curved sensor's response has a time constant of $35.3 \mu$ s. These results correspond to effective bandwidths of 11.21 and $4.51 \mathrm{kHz}$ for the straight and curved sensors, respectively. The greater volume of silicon that comprises the shaped sensor results in a higher thermal mass, meaning that the sensor is slower to respond to changes in heat flow than the straight sensor. For comparison, the same bias step change was applied to the straight and curved sensors with the length of $30 \mu \mathrm{m}$, as shown in Fig. 11(b). The responses have time constants of 10.7 and $24.4 \mu$ s for the straight and curved sensors, respectively. These correspond to bandwidths of 14.91 and $6.51 \mathrm{kHz}$ for the straight and curved sensors, respectively. As expected, the smaller thermal mass within these smaller sensors of length $30 \mu$ m makes them more responsive to changes in thermal heat flow.

Fig. 12 shows a comparison between the stage displacement measured by the MSA and the output of the $50-\mu \mathrm{m}$ curved electrothermal sensors when a step voltage is applied to the actuator of the nanopositioner. Due to the undamped resonant behavior of the stage, the step response is almost a singletone pure sinusoid within the first $2 \mathrm{~ms}$ after applying the step. Assuming a first-order low-pass model for the sensor, the sensor bandwidth can be calculated by measuring the phase difference between the actual stage displacement and the sensor output. As indicated in Fig. 12, the response of the sensor output has a phase delay of approximately $34^{\circ}$ with respect to the stage's measured displacement. Based on this phase shift and the frequency of the oscillations, the sensor bandwidth is calculated to be approximately $4.67 \mathrm{kHz}$, which matches quite well with the bandwidth of $4.51 \mathrm{kHz}$ previously calculated for this sensor.

Fig. 13 shows the drift in the outputs of the electrothermal sensors over a period of 2000 s, with the sensor biases being adjusted such that both sensors have equal sensitivities. During this time, the device was left in normal laboratory conditions, with no input being provided to the nanopositioner's actuators. For the curved sensors, the standard deviation from the mean output value was measured to be $4.76 \mathrm{mV}$, which corresponds to 


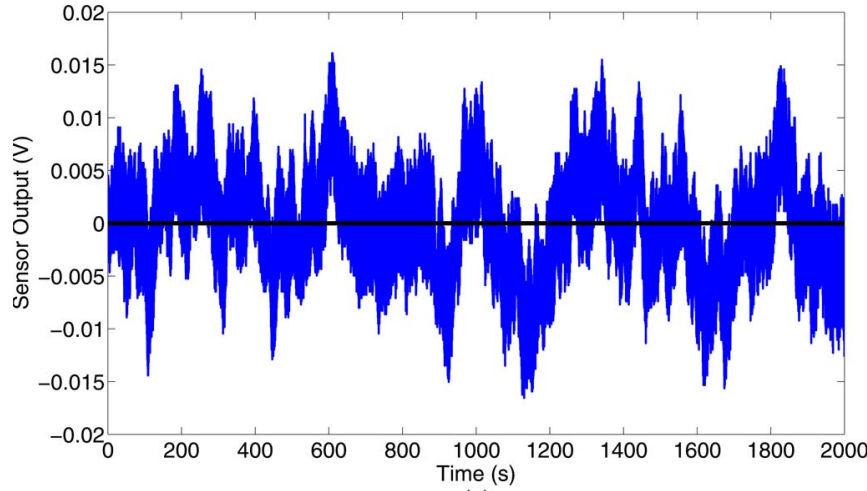

(a)

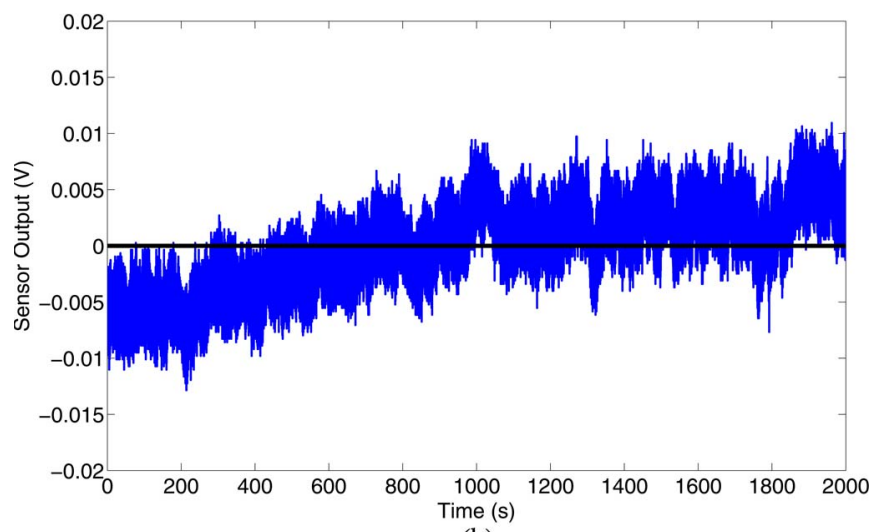

(b)

Fig. 13. Drift in output of 50- $\mu \mathrm{m}$ sensors. (a) Curved sensors. (b) Straight sensors.

a stage displacement of $13.95 \mathrm{~nm}$. In comparison, the standard deviation from the straight sensor's mean output was measured to be $3.73 \mathrm{mV}$, which corresponds to a displacement of $11.79 \mathrm{~nm}$. The higher standard deviation of the curved sensor output is largely due to the presence of low-frequency noise. This is not as prominent in the output of the straight sensor, which instead exhibits higher linear drift.

\section{CONCLUSION}

In this paper, a novel electrothermal sensor with a nonuniform heater profile has been introduced with the aim of improving sensor performance by creating a flatter temperature profile. The temperature distribution of the proposed heater shape was calculated and compared with that of a uniformly shaped heater, with simulation results confirming that a more spatially uniform temperature profile is achieved. Experimental testing shows that, with the same bias voltage, the shaped sensor design achieves a higher sensitivity than the conventional straight sensor. The linearities of the sensor designs are compared by analyzing the THD figures of the sensor outputs, with the figure of $4.43 \%$ measured using the shaped sensor being a significant improvement on the figure of $8.45 \%$ measured using the conventional sensor. In addition, the frequency-domain outputs show that the shaped sensor design possesses a lower noise floor than the conventional sensor. An analysis of the bandwidths of the sensors was performed by measuring the response of each sensor to a step change in applied bias voltage. These tests indicate that, due to the higher thermal mass of the shaped heaters, they possess a lower operating bandwidth compared to the uniformly shaped heaters. These results suggest that the design of electrothermal sensors for displacement measurement involves a number of tradeoffs between qualities such as sensor linearity, sensitivity, power consumption, and dynamic performance.

\section{ACKNOWLEDGMENT}

The scanning electron microscope images were obtained with the assistance of the Electron Microscope and X-Ray Unit at The University of Newcastle.

\section{REFERENCES}

[1] J. Gorman, Y. Kim, and N. Dagalakis, "Control of MEMS nanopositioners with nano-scale resolution," in Proc. ASME Int. Mech. Eng. Congr. Expo., 2006, pp. 151-159.

[2] L. L. Chu and Y. B. Gianchandani, "A micromachined 2D positioner with electrothermal actuation and sub-nanometer capacitive sensing," J. Micromech. Microeng., vol. 13, no. 2, pp. 279-285, Mar. 2003.

[3] M. A. Lantz, G. K. Binnig, M. Despont, and U. Drechsler, "A micromechanical thermal displacement sensor with nanometre resolution," Nanotechnology, vol. 16, no. 8, pp. 1089-1094, Aug. 2005.

[4] Y. Zhu, S. O. R. Moheimani, and M. R. Yuce, "Simultaneous capacitive and electrothermal position sensing in a micromachined nanopositioner," IEEE Electron Device Lett., vol. 32, no. 8, pp. 1146-1148, Aug. 2011.

[5] Y. Zhu, A. Bazaei, S. O. R. Moheimani, and M. R. Yuce, "Design, modeling, and control of a micromachined nanopositioner with integrated electrothermal actuation and sensing," J. Microelectromech. Syst., vol. 20, no. 3, pp. 711-719, Jun. 2011

[6] P. Vettiger, G. Cross, M. Despont, U. Drechsler, U. Durig, B. Gotsmann, W. Haberle, M. Lantz, H. Rothuizen, R. Stutz, and G. Binnig, "The 'millipede'-Nanotechnology entering data storage," IEEE Trans. Nanotechnol., vol. 1, no. 1, pp. 39-55, Mar. 2002.

[7] E. Eleftheriou, T. Antonakopoulos, G. Binnig, G. Cherubini, M. Despont, A. Dholakia, U. Durig, M. Lantz, H. Pozidis, H. Rothuizen, and P. Vettiger, "Millipede-A MEMS-based scanning-probe data-storage system," IEEE Trans. Magn., vol. 39, no. 2, pp. 938-945, Mar. 2003.

[8] M. Lantz, H. Rothuizen, U. Drechsler, W. Haberle, and M. Despont, "A vibration resistant nanopositioner for mobile parallel-probe storage applications," J. Microelectromech. Syst., vol. 16, no. 1, pp. 130-139, Feb. 2007.

[9] J. Chow and Y. Lai, "Displacement sensing of a micro-electro-thermal actuator using a monolithically integrated thermal sensor," Sens. Actuators A, Phys., vol. 150, no. 1, pp. 137-143, Mar. 2009.

[10] A. Mohammadi, M. R. Yuce, and S. O. R. Moheimani, "Dealing with 1/f noise in MEMS electrothermal sensing," in Proc. 37th IEEE Annu. Conf. IECON, Nov. 2011, pp. 4054-4058.

[11] A. Mohammadi, M. R. Yuce, and S. O. R. Moheimani, "Frequency modulation technique for MEMS resistive sensing," IEEE Sensors J., vol. 12, no. 8, pp. 2690-2698, Aug. 2012.

[12] U. Dauderstädt, P. de Vries, R. Hiratsuka, and P. Sarro, "Silicon accelerometer based on thermopiles," Sens. Actuators A, Phys., vol. 46, no. 1-3, pp. 201-204, Jan./Feb. 1995.

[13] G. Binnig, M. Despont, U. Drechsler, W. Häberle, M. Lutwyche, P. Vettiger, H. Mamin, B. Chui, and T. Kenny, "Ultrahigh-density atomic force microscopy data storage with erase capability," Appl. Phys. Lett., vol. 74, no. 9, pp. 1329-1331, Mar. 1999.

[14] A. Sebastian and D. Wiesmann, "Modeling and experimental identification of silicon microheater dynamics: A systems approach," J. Microelectromech. Syst., vol. 17, no. 4, pp. 911-920, Aug. 2008.

[15] A. Pantazi, A. Sebastian, G. Cherubini, M. Lantz, H. Pozidis, H. Rothuizen, and E. Eleftheriou, "Control of MEMS-based scanningprobe data-storage devices," IEEE Trans. Control Syst. Technol., vol. 15, no. 5, pp. 824-841, Sep. 2007.

[16] Y. Zhu, A. Bazaei, S. O. R. Moheimani, and M. R. Yuce, "A micromachined nanopositioner with on-chip electrothermal actuation and sensing," IEEE Electron Device Lett., vol. 31, no. 10, pp. 1161-1163, Oct. 2010.

[17] Y. Zhu, A. Bazaei, S. O. R. Moheimani, and M. R. Yuce, "Design, prototyping, modeling and control of a MEMS nanopositioning stage," in Proc. ACC, Jun. 29-Jul. 1, 2011, pp. 2278-2283. 
[18] A. Bazaei, Y. Zhu, S. O. R. Moheimani, and M. R. Yuce, "Analysis of nonlinear phenomena in a thermal micro-actuator with a built-in thermal position sensor," IEEE Sensors J., vol. 12, no. 6, pp. 1772-1784, Jun. 2012.

[19] B. Krijnen, R. P. Hogervorst, J. W. van Dijk, J. B. C. Engelen, L. A. Woldering, D. M. Brouwer, L. Abelmann, and H. M. J. R. Soemers, "A single-mask thermal displacement sensor in MEMS," J. Micromech. Microeng., vol. 21, no. 7, p. 074007, Jul. 2011.

[20] U. Dürig, "Fundamentals of micromechanical thermoelectric sensors," J. Appl. Phys., vol. 98, no. 4, pp. 044906-1-044906-14, Aug. 2005.

[21] A. Sebastian, "Systems and control approach to electro-thermal sensing," in Control Technologies for Emerging Micro and Nanoscale Systems, E. Eleftheriou and S. O. R. Moheimani, Eds. New York, NY, USA Springer-Verlag, 2011, ch. 8, pp. 137-152.

[22] S.-C. Chen and M. L. Culpepper, "Design of contoured thermomechanical actuators and pulsing actuation to enhance dynamic performance," J. Microelectromech. Syst., vol. 21, no. 2, pp. 340-349, Apr. 2012.

[23] S. Chen and M. Culpepper, "Design of contoured microscale thermomechanical actuators," J. Microelectromech. Syst., vol. 15, no. 5, pp. 12261234, Oct. 2006.

[24] A. Kwan, S. Song, X. Lu, L. Lu, Y. Teh, Y. Teh, E. Chong, Y. Gao, W. Hau, F. Zeng, M. Wong, C. Huang, A. Taniyama, Y. Makino, S. Nishino, T. Tsuchiya, and O. Tabata, "Designs for improving the performance of an electro-thermal in-plane actuator," in Proc. IEEE/IFIP 19th Int. Conf. VLSI-SoC, Oct. 2011, pp. 220-225.

[25] A. M. H. Kwan, S. Song, X. Lu, L. Lu, Y. K. Teh, Y. F. Teh, E. W. C. Chong, W. Gao, Y. Hau, F. Zeng, M. Wong, C. Huang, A. Taniyama, Y. Makino, S. Nishino, T. Tsuchiya, and O. Tabata, "Improved designs for an electrothermal in-plane microactuator," J. Microelectromech. Syst., vol. 21, no. 3, pp. 586-595, 2012.

[26] A. Cowen, G. Hames, D. Monk, S. Wilcenski, and B. Hardy, SOIMUMPs Design Handbook (Revision 8.0). Durham, NC, USA: MEMSCAP, Inc., 2011.

[27] R. Legtenberg, A. W. Groeneveld, and M. Elwenspoek, "Comb-drive actuators for large displacements," J. Micromech. Microeng., vol. 6, no. 3, pp. 320-329, Sep. 1996

[28] K. Laszczyk, S. Bargiel, C. Gorecki, J. Krężel, P. Dziuban, M. Kujawińska, D. Callet, and S. Frank, "A two directional electrostatic comb-drive X-Y micro-stage for MOEMS applications," Sens. Actuators A, Phys., vol. 163, no. 1, pp. 255-265, Sep. 2010.

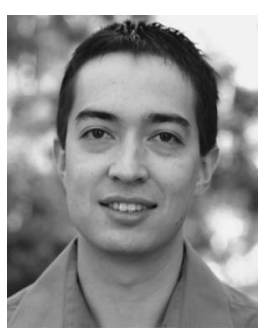

Anthony G. Fowler (S'10) was born in Taree, Australia. He received the B.S. degree in electrical engineering from The University of Newcastle, Callaghan, Australia, in 2010, where he is currently working toward the Ph.D. degree in electrical engineering.

His research interests include the design and analysis of novel microelectromechanical-systems devices for energy harvesting and nanopositioning applications.

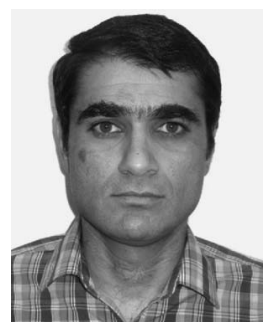

Ali Bazaei (M'10) received the B.Sc. and M.Sc. degrees in electrical engineering from Shiraz University, Shiraz, Iran, in 1992 and 1995, respectively, the $\mathrm{Ph} . \mathrm{D}$. degree in electrical engineering from Tarbiat Modares University, Tehran, Iran, in 2004, and the $\mathrm{Ph} . \mathrm{D}$. degree in electrical engineering from the University of Western Ontario, London, ON, Canada, in 2009.

From September 1995 to January 2000, he was an Instructor with Yazd University, Yazd, Iran. From September 2004 to December 2005, he was a Research Assistant with the Department of Electrical and Computer Engineering, University of Western Ontario, where he commenced a Ph.D. program in January 2006. Since April 2009, he has been a Postdoctoral Researcher with the School of Electrical Engineering and Computer Science, The University of Newcastle, Callaghan, Australia. His research interests lie in the general area of nonlinear systems, including control and modeling of structurally flexible systems, friction modeling and compensation, and neural networks.

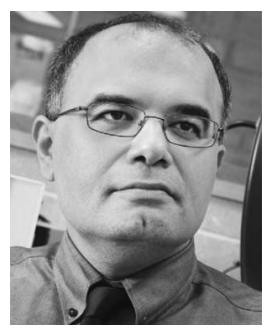

S. O. Reza Moheimani (M'97-SM'00-F'11) received the B.Sc. degree in electrical and electronic engineering from Shiraz University, Shiraz, Iran, in 1990 and the M.Eng.Sc. and Ph.D. degrees in electrical and electronic engineering from the University of New South Wales, at the Australian Defence Force Academy, Canberra, Australia, in 1993 and 1996, respectively.

$\mathrm{He}$ joined The University of Newcastle, Callaghan, Australia, in 1997, where he is a Professor of Electrical Engineering and an Australian Research Council (ARC) Future Fellow. He is the founder and Director of the Laboratory for Dynamics and Control of Nanosystems, a multimillion-dollar state-of-the-art research facility. During 2003-2010, he was the Associate Director of the ARC Centre of Excellence for Complex Dynamic Systems and Control. His current research interests are mainly in the area of ultrahigh-precision mechatronic systems, with particular emphasis on dynamics and control at the nanometer scale, including applications of control and estimation in nanopositioning systems for high-speed scanning probe microscopy, modeling and control of microcantilever-based devices, control of microactuators in microelectromechanical systems, and control issues related to ultrahigh-density probe-based data-storage systems.

Prof. Moheimani is a Fellow of the International Federation of Automatic Control (IFAC) and the Institute of Physics (U.K.). He is a corecipient of the 2007 IEEE TRansactions on Control Systems TeChnology Outstanding Paper Award and the 2009 IEEE Control Systems Technology Award - the latter with a group of researchers from IBM Zurich Research Labs, where he has held several visiting appointments. He has served on the editorial boards of a number of journals, including the IEEE/ASME TRANS ACTIONS ON MECHATRONICS, IEEE TRANSACTIONS ON CONTROL SYSTEMS TECHNOLOGY, and Control Engineering Practice. He has chaired several international conferences and workshops and currently chairs the IFAC Technical Committee on Mechatronic Systems. 